\title{
Simulation and experiments on friction and wear of diamond: a material for MEMS and NEMS application
}

\author{
Tahir Çağın $\dagger$, Jianwei Che $\dagger$, Michael N Gardos $\ddagger$, Amir Fijany§ and \\ William A Goddard III $\dagger$
}

$\dagger$ Materials and Process Simulation Center, 139-74, California Institute of Technology, Pasadena, CA 91125, USA

\$ Raytheon, Communication Systems, El Segundo, CA 90245, USA

$\S$ Jet Propulsion Laboratory, NASA, Pasadena, CA 9100, USA

Received 22 January 1999

\begin{abstract}
To date most of the microelectromechanical system (MEMS) devices have been based on silicon. This is due to the technological know-how accumulated on the manipulation, machining and manufacturing of silicon. However, only very few devices involve moving parts. This is because of the rapid wear arising from high friction in these silicon-based systems. Recent tribometric experiments carried out by Gardos on silicon and polycrystalline diamond (PCD) show that this rapid wear is caused by a variety of factors, related both to surface chemistry and cohesive energy density of these likely MEMS bearing materials. In particular, the 1.8-times stronger $\mathrm{C}-\mathrm{C}$ bond in diamond as opposed to the $\mathrm{Si}-\mathrm{Si}$ bond in the bulk translates into a more than $10^{4}$-times difference in wear rates, even though the difference in flexural strength is only 20-times, in hardness 10-times and in fracture toughness 5-times. It also has been shown that the wear rates of silicon and PCD are controlled by high-friction-induced surface cracking, and the friction is controlled by the number of dangling, reconstructed or adsorbate-passivated surface bonds. Therefore, theoretical and tribological characterization of Si and PCD surfaces is essential prior to device fabrication to assure reliable MEMS operation under various atmospheric environments, especially at elevated temperatures.

As a part of the rational design and manufacturing of MEMS devices containing moving mechanical assemblies (MEMS-MMA) and especially nanoelectromechanical devices (NEMS), theory and simulation can play an important role. Predicting system properties such as friction and wear, and materials properties such as thermal conductivity is of critical importance for materials and components to be used in MEMS-MMAs. In this paper, we present theoretical studies of friction and wear processes on diamond surfaces using a steady state molecular dynamics method. We studied the atomic friction of the diamond-(100) surface using an extended bond-order-dependent potential for hydrocarbon systems. Unlike traditional empirical potentials, bond order potentials can simulate bond breaking and formation processes. Therefore, it is a natural choice to study surface dynamics under friction and wear. In order to calculate the material properties correctly, we have established a consistent approach to incorporate non-bond interactions into the bond order potentials. We have also developed an easy-to-use software to evaluate the atomic-level friction coefficient for an arbitrary system, and interfaced it into a third-party graphical software.
\end{abstract}

\section{Introduction}

Microelectromechanical systems (MEMS) is an emerging enabling technology that merges advances in information processing, storage, and display with advances in sensors and actuators to bring about a revolution in the way we perceive and control the environment [1-7].

MEMS is a natural progression in the capabilities of semiconductor devices. The ability of MEMS to gather and process information, decide on a course of action, and control the environment through actuators increases the affordability, functionality, and number of smart systems. A very significant role is attributed to MEMS for the technologies of the 21 st century, as applied to the following areas:

- optical switches and aligners

- parts handling in manufacturing

- inertial navigation

- pumps and valves 
- data storage

- computer display

- structural control of aircrafts.

The design of MEMS presents challenges at various lengths and timescales. The length scale ranges from angstroms to millimetres where theories such as quantum mechanics, classical mechanics, solid (continuum) mechanics may be employed to improve practical performance.

Most of the MEMS devices explored and designed to date are based on silicon, due to technological knowhow accumulated on this particular semiconductor with regard to manipulating, machining, manufacturing etc on the microscale. Recent tribometric experiments carried out at Hughes by Gardos [8-11] strengthen the hypothesis that the magnitude of adhesion and thus the adhesive friction between silicon and diamond surfaces are essentially defined by the number of dangling (high-friction), reconstructed (reduced-friction) or adsorbate-passivated (low-friction) surface bonds. Incipient linking of the sliding counterfaces by unsaturated (dangling) bonds on heating and their passivation by benign, chemisorbed gases (e.g., hydrogen) were suggested as the primary causes of radically increased and reduced adhesion and friction, respectively. Hence to a performance degradation in silicon-based MEMS through a higher sliding friction and a resulting short wear life for devices with moving parts.

With this observation one must look into alternative materials, such as single crystal and polycrystalline diamond (PCD) where sliding friction measurements lead to considerably lower values. The wear rate of PCD is about four orders of magnitude lower than those of variety of $\mathrm{Si}$ crystallinites (poly-Si, $\mathrm{Si}(100)$ and $\mathrm{Si}(111)$ ). In designing the next generation MEMS using PCD, there is a lot to be done. The hypothesis brought forth by Gardos to explain his experimental results on the basis of the chemical state of the surface bonds needs to be explored and rationalized theoretically.

Replacing conventional devices with their MEMS/ NEMS analogues presents both opportunities and challanges. The evolution of small machines and sensors demonstrates that ever-increasing number of these devices with electronic circuits will yield a window to the world of motion, sound, heat and other physical forces. The current MEMS devices contain about 10 components MEMS devices. The anticipated applications such as pumps and valves require $10^{3}-10^{5}$ components, optical aligners may have $10^{2}-10^{4}$ components. In computer displays this range goes up to $10^{6}$ or $10^{7}$. The design, processing and fabrication methodology for such applications requires research not only in the materials area but also into the operational conditions of such multi-component systems, where simulation codes developed for robotics application will play a critical role [15]. Simulation of systems with such a large number of components will require the use of highly optimized parallel programs which we aim at developing and testing through MEMS applications.

\subsection{Problem of friction and wear}

Friction is an unavoidable, parasitic force in flea-powered MEMS-MMAs. It is a crucial factor that determines not only efficiency but durability. Gardos' SEM-tribometric data in [8-13] indicated that high adhesive friction within the Hertzian contact zones of both silicon and PCD causes shearinduced microcracking in the wake of the rolling/sliding contact. The ensuing wear and tolerance losses alone will fail these devices, especially when they are fabricated from high friction and high wear rate bearing materials such as silicon. Although the hardness and chemical stability of PCD renders it a difficult material to shape into MEMSMMA components and assembly of these components into complex micromachinery is even more difficult than those made of silicon, the properties of PCD are far better for MEMS-MMA applications. Diamond is the hardest material known in nature, with a surprisingly high strength and fracture toughness in its polycrystalline form. Its thermal conductivity is high, readily dissipating both frictional and microelectronics-generated heat from functional areas. Due to the higher strength of the covalent carbon-hydrogen bonds, as compared with that of silicon-hydrogen bonds, hydride-passivated PCD surfaces remain lubricative at higher operating temperatures.

It is important to understand that bench-level testing in the laboratory is useful only if the behaviour of real devices is closely approximated. Theory becomes a problem-solving tool only if it accurately mimics practice. For example, the high friction and rapid wear of a silicon rotor in an electrostatic micromotor is better approximated by SEM tribometry under realistic Hertzian stresses than by the action of a sharp atomic force microscope (AFM) silicon (wearless) tip brought only close to a highly flat silicon surface. Employing the concept of a reactive hydrocarbon potential, the molecular dynamics simulation technique described in the present paper is able to study both the 'wearless' (AFM-measured) friction forces at the atomic level, as well as the coefficients of friction (COF) measured under full Hertzian (engineering) contact conditions. As an added benefit, the reactive potential permits the study of lubricative surface adsorbates. Therefore, simulation of MEMS-MMA interfaces can not only rationalize tribometric results, but provide detailed information on contact kinematics under artificially induced surface chemical conditions. The various likely fabrication processes can also be iterated before benchlevel experiments are attempted.

\section{Experiments}

\subsection{The SEM tribometer and test procedures}

As shown schematically in figure 1, a small flat is oscillated against a hemispherically tipped and dead-weight-loaded pin, either in the $1.33 \times 10^{-3} \mathrm{~Pa}\left(1 \times 10^{-5}\right.$ Torr $)$ vacuum of the SEM column or in a lidded and preferentially pumped Knudsen cell-like subchamber backfilled and continually purged with selected, high-purity gases (e.g., air, $\mathrm{H}_{2}, \mathrm{He}$, $\mathrm{N}_{2}$ and $\mathrm{O}_{2}$ ) introduced from lecture bottles.

The stage housing the heated flat is oscillated at $0.5 \mathrm{~Hz}$, with a small average sliding speed of $3.5 \mathrm{~mm} \mathrm{~s}^{-1}$. The Hertzian contact stresses are applied by normal (dead weight) loads on the pin, ranging from 6 to $50 \mathrm{~g}(0.06-0.49 \mathrm{~N})$. The flat may be held at room temperature or heated and 


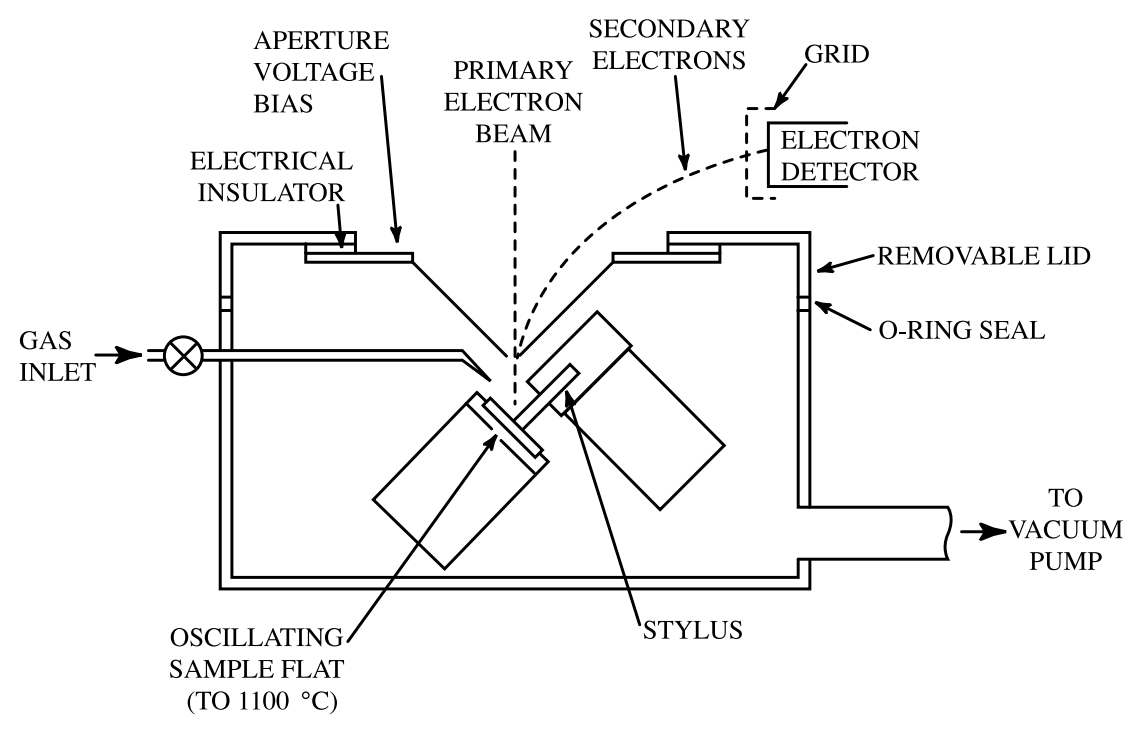

Figure 1. The schematic of the SEM tribometer.

cooled to and from $1100^{\circ} \mathrm{C}(\max )$ at various thermal ramping rates. The friction forces are archived and converted in real time to averaged coefficients of friction (COF) by a desktop computer, using commercial data logging and analysis software. A more thorough description of the apparatus and procedures for examining $\mathrm{PCD}$ and $\mathrm{Si}$ in vacuum may be found in [14]. The equivalent methods for testing a variety of materials in partial pressures of $\mathrm{H}_{2}$ and $\mathrm{O}_{2}$ were previously described in [8,9] and [11], respectively. A constantly exchanged gas environment maintained at a given partial pressure offers several benefits to testing PCD. Several thermally ramped experiments could be repeated without breaking either the SEM column vacuum or the particular test atmosphere, because the extremely low wear of diamond precluded the need to photograph the wear scars after each test. Furthermore, the high thermal conductivity of the gases (compared with vacuum) allows cooling of the friction force strain gages to near RT in a reasonably short period of time between tests.

The SEM tribometer has no in situ surface analytical capability. Nevertheless, the extensive Si and PCD work by Gardos in [8-12] and related data from the literature has allowed some interpretation of the friction trends as a function of temperature and atmosphere in terms of atomic-level surface behaviour. The magnitude of adhesion and thus the shape and magnitude of the SEMtribometric COF curves in were defined by the number of dangling (high-friction), reconstructed (reduced-friction) or adsorbate-passivated (low-friction) surface bonds. Incipient linking of the sliding counterfaces by unsaturated bonds on heating and their deactivation by benign adsorbates on cooling were suggested as the primary causes of radically increased and reduced adhesion and friction, respectively.

Even if the SEM tribometer had in situ surface analytical capability or the analyses of the wear scars were attempted after the experiments in vacuum and partial pressures of hydrogen, commonly employed techniques such as Auger electron spectroscopy (AES) and x-ray photoelectron spectroscopy (XPS) are not sensitive to surface hydrides. Infrared spectroscopy is able to show hydrogen, oxygen or hydroxyl moieties covalently bonded to carbon, but lacks sensitivity for an essentially monolayer coverage (or less in the case of surface reconstruction). Electron energy loss spectroscopies require conductive samples of high surface finish to avoid charging effects and increase electron reflectivity at low electron energies. Since wear scars are still rough on the microscopic scale and any exposure to atmospheric air leads to some contamination with adventitious carbon, this analytical technique becomes problematic with tribospecimens. Surface charging is higher with increased purity (i.e. progressively lower $\mathrm{sp}^{2}$ content) and causes shifts in the AES and XPS energy peaks. Where the wear scar is much smaller than the diameter of the probing XPS beam, the chemical state of the worn surface cannot be determined with certainty.

As a consequence, explaining any kind of interaction at the atomic level via macroscopic friction measurements could only be validated by testing a large number of $\mathrm{Si}$ and PCD crystal structures with the highest possible crystallographic and environmental control in vacuum and in partial pressures of hydrogen, and watching for any changes in the characteristic friction and wear trends. To date, the results and the related literature data have helped to explain the high torque and wear rate of miniaturized tribomechanical parts made from $\mathrm{Si}$, why molecular hydrogen can act as an atomic-level lubricant for both Si and PCD, and why PCD is a better bearing material for dynamic MEMS-MMAs than Si.

\subsection{SEM-tribometric results}

As more thoroughly described by Gardos in [8-14], typical SEM-tribometric coefficient of friction (COF) trends with PCD and silicon interfaces heated and cooled in vacuum 
and hydrogen are exemplified by the unusual, 'rabbit-earlike' friction curves shown in figure 2. These typical COF functions contain a great deal of conjectured surface chemical information, but are yet to be confirmed by independent experimentation or rationalized by theoretical simulation.

As depicted in figure 2, the substantial increase in COF $\mathrm{PCD}$ and COF Si at some elevated temperature is attributed to the generation of dangling surface bonds on the desorption of adsorbates combined with wear-induced tearing of the counterfaces. There is a shift in the COF-increase peaks to higher temperatures going from vacuum to low partial pressures of hydrogen test atmospheres. This shift is in accordance with the LeChâtelier principle: if a reaction (the desorption of an adsorbate) results in a gas (e.g. $\mathrm{H}_{2}$ ) as a product, increasing the partial pressure (activity) of this gas in the tribometer chamber will retard the reaction, i.e. desorption will occur at higher temperatures [9].

The substantial reduction in the COF PCD on cooling in vacuum or in hydrogen is the footprint of tribocatalytically enhanced chemisorption of water (i.e. the residual atmospheric moisture in the SEM column) or molecular hydrogen purposely introduced into the test chamber. There is also some reduction in the COF Si in vacuum on cooling, attributed to the chemisorption of residual water vapour in the SEM column. However, the final friction values near room temperature were not as low as the starting COF, as explained by the large increase in the apparent areas of contact resulting from the high wear rate of $\mathrm{Si}$ (some $10^{4}$-times greater than those of PCD).

In contrast, the final $\mathrm{COF} \mathrm{Si}$ in hydrogen at room temperature was consistently and significantly higher than in vacuum. Although this different trend is consistent with the lack of activation energy for the chemisorption of $\mathrm{H}_{2}$ on cooling [9], it is difficult to believe that surface bonds remain unsaturated at or near room temperature in the presence of hydrogen. If this phenomenon is not caused by unpassivated free radicals but by the interaction of variously reconstructed surfaces with energies changing as a function of temperature in the presence of adsorbates [10-14], the SEM tribometer is not able to separate these variables. The trough-like 'bathtub' (dip-and-rise) curve in the COF at the highest temperatures, both in vacuum and in rarefied hydrogen, can be attributed to surface re(de)construction. Continued heating of the progressively degased and worn surfaces reconstructs the dangling bonds to reduce the surface energy, apparently unhindered by the ongoing tribological action. Since the worn and crystallographically always miscut surfaces display crystallographic disorder, a variety of reconstruction cells can be formed. However, the real area of contact is still extremely large relative to pi-chain dimensions. It follows, therefore, that a global reduction in COF may be explained by a local decrease in the surface energy within each nanoscaled reconstruction mosaic.

Once the heating stops and the rubbed surfaces cool below a certain temperature, the activation energy needed to keep the bonds reconstructed is lost. There is a significant rise in friction from the deepest part of the COF trough due to regeneration of the dangling bonds (deconstruction), commensurate with an increase in surface energy (another COF peak). As the contact is cooled further to the temperature where adsorbates readily chemisorb and passivate the deconstructed surface bonds, the lowest possible surface energy and a large reduction in COF are attained.

The details of this typical friction trend characteristic to both PCD and the silicon crystal structures (e.g. poly$\mathrm{Si}, \mathrm{Si}(100)$ and $\mathrm{Si}(111)$ ) tested lend themselves well to theoretical treatment by $a b$ initio [19] and molecular dynamics calculations.

\section{Theoretical background}

Molecular dynamics have been widely used to study structures and properties of a variety of substances. It provides information on the atomic level directly. Therefore, people can test their theories and explain the experimental results on the basis of atomic motion. In molecular dynamics simulation, one of the most important steps is to apply a proper empirical potential to the system of interest. For a system with a given initial condition, its subsequent dynamics are completely determined by the potential surface that it evolves on. Choosing a proper classical potential is crucial to reproduce the real physical behaviour of the system.

Usually, friction is accompanied by wear. Although it is possible to have wearless friction at atomic level, we would like to have a potential which is applicable under both conditions for consistency. In our study, we employ a modified bond order hydrocarbon potential [16-18]. It allows chemical reactions during dynamics, therefore, it is a natural candidate to simulate both wearless friction and general friction. Our modified Brenner bond order potential energy is written as,

$$
V_{t o t}=\sum_{i} \sum_{j>i}\left[V^{B}\left(r_{i j}\right)+V^{N B}\left(r_{i j}\right)\right],
$$

where

$$
\begin{gathered}
V^{B}\left(r_{i j}\right)=V_{R}\left(r_{i j}\right)+\bar{B}_{i j} V_{A}\left(r_{i j}\right), \\
V^{N B}\left(r_{i j}\right)=P(i, j) V^{L R}\left(r_{i j}\right) .
\end{gathered}
$$

In the above equations, $V^{B}$ is a bond term, $V^{N B}$ is a nonbond term; $V_{R}$ and $V_{A}$ are the repulsive and attractive portion of a general Morse potential, respectively; $B_{i j}$ is the bond order parameter; $P(i, j)$ is a screening function which considers the nonbond contribution properly and $V^{L R}$ is a general notation for nonbond interaction, such as van de Waals or Coulomb forces.

\subsection{Simulation results}

With the modified bond order potential, we study the friction process on reconstructed diamond-(100) surfaces. Both bare and hydrogenated surfaces are investigated. In our simulation, two diamond crystals are put in contact. The bottom two layers of the lower block are held still, a constant velocity is assigned to the top two layers of the upper block. The external forces that are required to maintain the steady motion are recorded. As a result, the ratio between the external driving force and the normal force gives the friction coefficient. In order to prevent the system from heating up, a stochastic thermal bath is applied to maintain the average kinetic energy. 

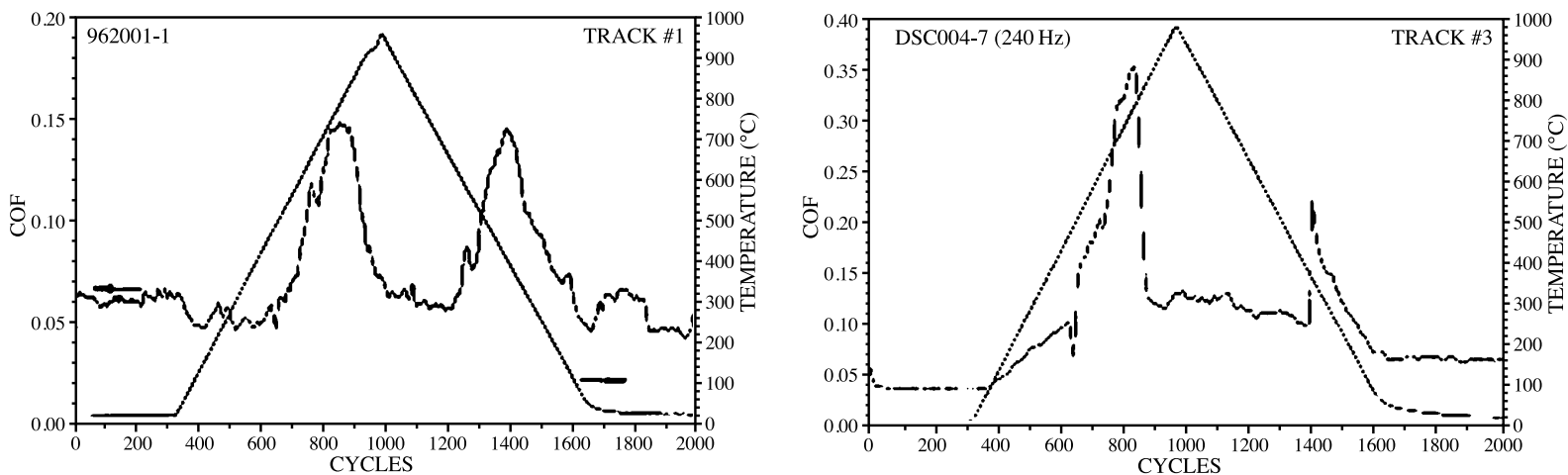

Figure 2. Friction profile for two different tracks display 'rabbit-ear-like' curves.

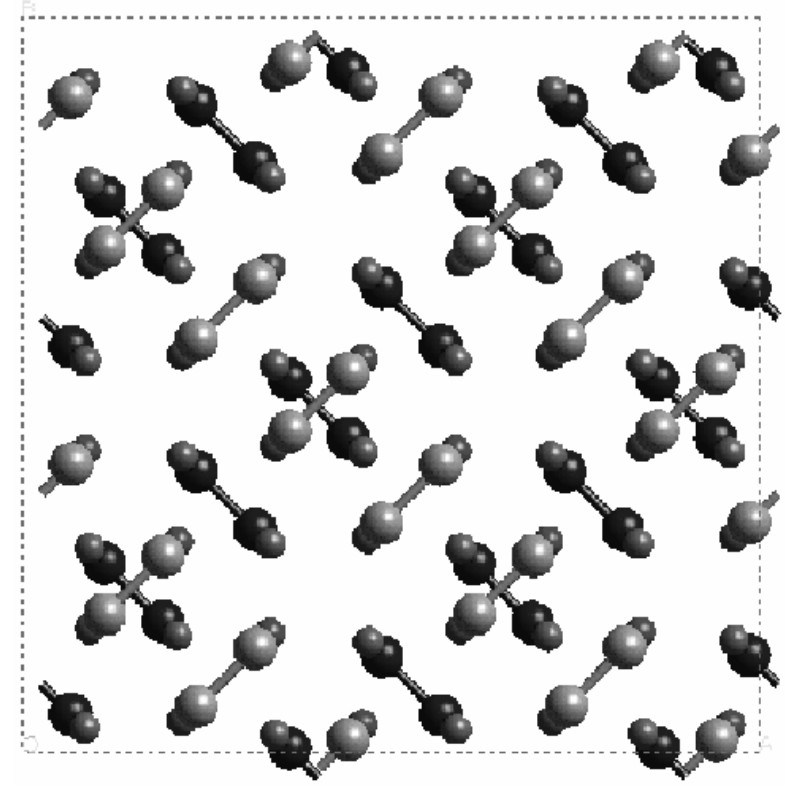

Figure 3. The top view of hydrogenated-(100) surfaces of two diamond crystals. Only the atoms at the interface are shown here. The grey and blue atoms are carbon atoms of moving block and fixed block, respectively. The red and green atoms are hydrogen atoms of upper and lower surfaces, respectively.

(This figure can be viewed in colour in the electronic version of the article; see www.iop.org)

In figure 3, we show the top view of two hydrogenated diamond-(100) surfaces. Both surfaces are C $2 \times 1$ reconstructed. However, they are placed at $90^{\circ}$ with respect to each other. The atoms on each surface create the surface potential with mountains and valleys. One can expect that different friction forces might arise from different movement on the microscopic level. The general experimental friction coefficients are the average value of many different microscopic results. In particular, the friction of PCD is an average quantity of friction along many different crystal orientations. In our simulation, we are able to extract each individual friction coefficient for each direction on a specific surface. We call this the differential friction coefficient. For the system shown in figure 3, we have calculated the differential friction for three different sliding directions. The three characteristic directions are the $x$ direction, $y$-direction, and $x y$-direction. Obviously, sliding

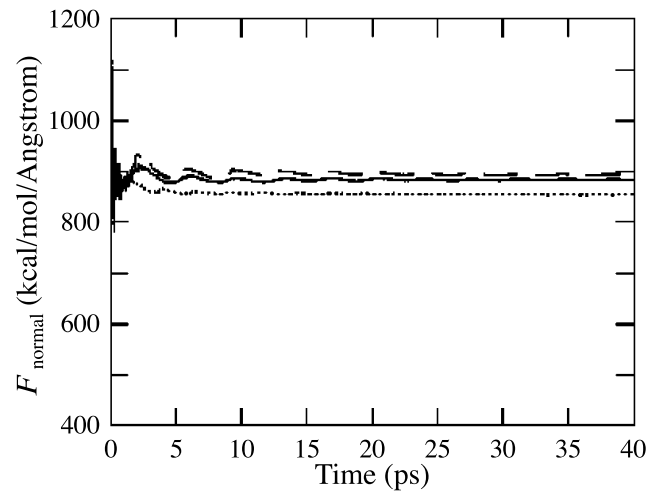

Figure 4. Running average of normal forces. The force from $x$-direction movement, solid curve; the force from $y$-direction movement, dashed curve and the force from $x y$-direction movement, dotted curve.

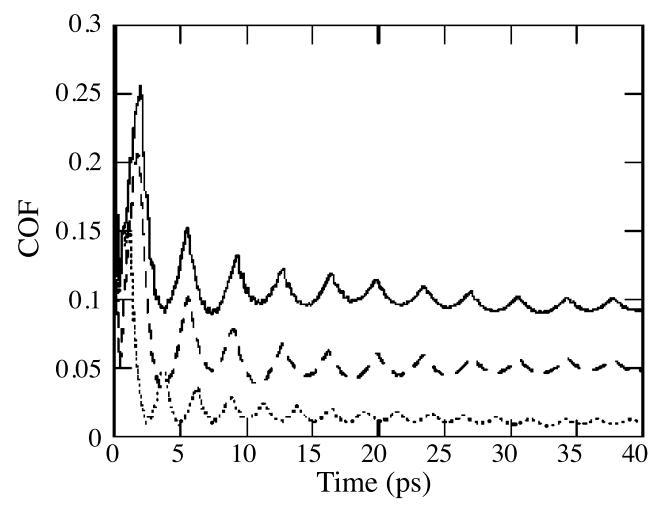

Figure 5. Running average of differential friction coefficients. The solid curve shows the $x$-direction friction coefficient, the dashed curve shows the $y$-direction friction coefficient, and the dotted curve shows the $x y$-direction friction coefficient.

along the $x y$-direction will have lowest resistance, because the path is along a potential valley. On the other hand, moving in the $x$-direction or $y$-direction has to climb the potential barriers created by the presence of hydrogen atoms along the path.

In figure 4 , we show the running averages of forces in the normal direction with respect to the movement. The initial oscillations represent the process of reaching a steady state motion. Usually, the system arrives at the steady state within $10 \mathrm{ps}$. The constant velocity is maintained at 


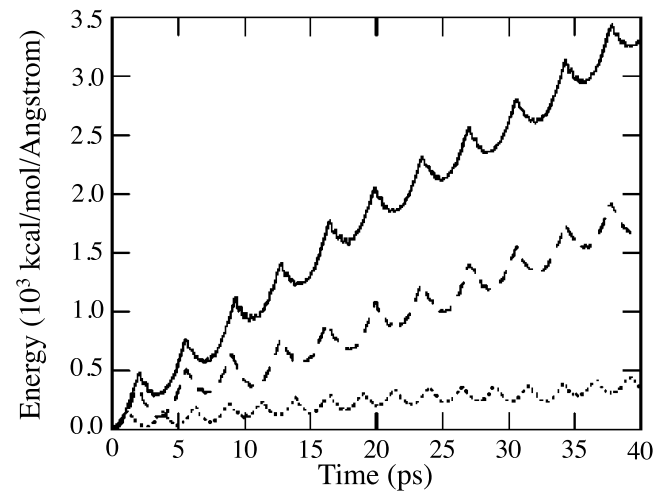

Figure 6. External work done to the system as a function of time. The solid curve is the work done while the top slab is moving along the $x$-direction, the dashed curve is the work done as the top slab is moving along the $y$-direction, and the dotted curve is the work done as the slab is moving along the diagonal in the $x y$-plane.

$1 \AA \mathrm{ps}^{-1}\left(100 \mathrm{~m} \mathrm{~s}^{-1}\right)$. Figure 4 illustrates that the normal forces are very similar despite different moving directions. This result indicates that any difference in the differential friction coefficients can only come from different motion arrangements in the regime of our investigation. Our normal force is around $850 \mathrm{kcal}^{-1} \mathrm{~mol}^{-1}$. Figure 5 depicts the running average of the friction coefficients calculated for each sliding direction. As we expected, the $x y$-direction has lowest friction coefficient, and the $x$-direction and $y$ direction have high friction coefficients. If the two surfaces are perfectly aligned, the $x$-direction and $y$-direction would have same friction coefficient. However, in our simulation, the model is set up such that the top block is shifted in the $y$-direction, to create a slightly different potential energy surface. In turn, this gives rise to a smaller friction coefficient in the $x$-direction.

One phenomenon that is always accompanied by friction is energy dissipation. The friction coefficient can also be extracted from the energy consumption curve. In figure 6, we plot the external work done to the system as a function of time. The slope of each curve gives the friction coefficient, and the detailed features of each curve reflect the potential surface contour along the each slide path. For instance, the three peaks in each period of the $x y$-direction curve represent each hydrogen atom on the lower surface passing through three reconstructed $\mathrm{C}-\mathrm{C}$ bonds of the upper surface.

In general, clean crystal surfaces are less stable because of the dangling bonds. This is also the case for diamond. The pi-chain reconstructed diamond-(100) surface is known to be energetically favourable. However, when two such surfaces are brought together, they can easily react, which increases the friction force more than two orders of magnitude. In this case, frictional force comes not only from nonbond interaction, but from chemical binding forces also. This is the main cause of large frictional force. Figure 7 shows the running average of the friction coefficient and the external work done on the system. Compared with the hydrogenated surface, the friction coefficient is much larger. Our simulation reveals the cost of formation and breaking of chemical bonds at the interface as displayed in figure 8 .

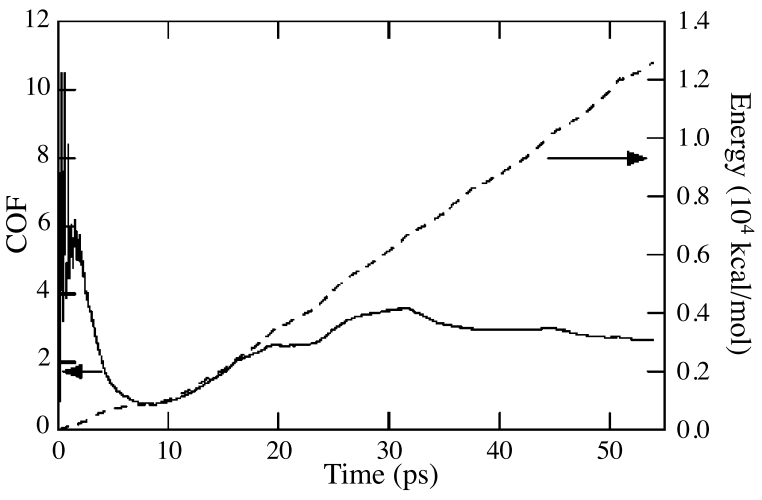

Figure 7. The running average of a differential friction coefficient and external work as a function of time.

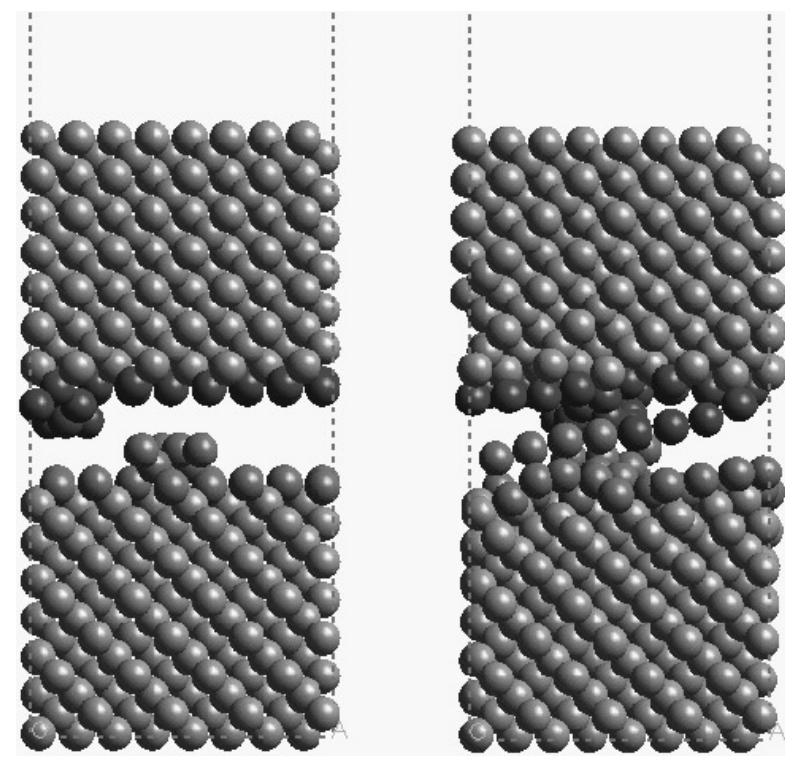

Figure 8. The initial and final structures of two diamond crystals sliding with respect to each other. The red and blue atoms represent the surface atoms.

(This figure can be viewed in colour in the electronic version of the article; see www.iop.org)

\section{Discussions and conclusion}

Friction and wear are complicated physical and chemical processes. Our investigation mainly focused on the atomic scale origin of friction. Crystal surfaces sliding in different directions gives different friction coefficients. The surface potential energy landscape (which is periodic for single crystal diamond) is the main source of this. Also, dangling bonds on surfaces can create a large frictional force. In this case, it is necessary to break these bonds in order to have relative movement. Our calculations clearly show how these aspects contribute to atomic friction and wear. In all cases, surface passivation has to be used to achieve low friction and wear rate. If possible, a specific sliding direction is preferred. Another way of decreasing friction forces is to add proper lubricants to the interface. Gardos [11] has reported that water is a perfect lubricant for both diamond and silicon. It also needs to be noted that there are other factors giving rise to the overall frictional force. For instance, surface 
T Çă̆ın et al

asperities and contact area can dramatically modify total friction coefficients.

The modified bond order potential is capable of dealing with both wearless friction and general friction. For this theoretical study, we have developed an easy-to-use software that can calculate the friction coefficient for a given system [20] and interfaced to a graphical molecular modelling package [21] using software developers kit (SDK) [22]. This program can also simulate the frictional process between various types of surfaces (lubricated or not). It can be used by experimenters as a guide to optimizing lubricants and studying the behaviour of these lubricants. This is extremely important for the design and further modification of new mechanical systems and lubricants.

\section{Acknowledgments}

This research was funded by a grant from NASA on computational nanotechnology. MNG acknowledges funding from the AFOSR tribology program. The facilities of the MSC are also supported by grants from NSF (ASC 9217368 and CHE 91-12279), ARO (MURI), ARO (DURIP), ONR (DURIP), Chevron Petroleum Technology Co., Asahi Chemical, Owens-Corning, Exxon, Chevron Chemical Co., Asahi Glass, Chevron Research Technology Co., Avery Dennison, BP America, and Beckman Institute.

\section{References}

[1] Gabriel K J 1995 Engineering microscopic machines Sci. Am. 273118

[2] Howe R T, Muller R S, Gabriel K J and Trimmer W S N 1990 Silicon micromechanics: sensors and actuators on a chip IEEE Spectrum (July) 2729

[3] Core T A, Tsang W K and Sherman S J 1993 Fabrication technology for an integrated surface-micromachined sensor Solid State Technol. (October) 3639
[4] Scott W B 1993 Micro-machines hold promise for aerospace Aviat. Week Space Technol. (March)

[5] Carey J and Gross N 1993 Mighty mites hit it big Business Week (April)

[6] Stix G 1992 Micron machinations Sci. Am. (November) 267 106

[7] Younsee J M 1993 Mirrors on a chip IEEE Spectrum (November) 3027

[8] Gardos M N 1996 Surface chemistry-controlled tribological behaviour of Si and diamond Tribol. Lett. 2173

[9] Gardos M N 1996 Tribol. Lett. 2355

[10] Gardos M N 1998 Re(de)construction-induced friction signatures of polished polycrystalline diamond films in vacuum and hydrogen Tribol. Lett. 4175

[11] Gardos M N 1997 Protective coatings and thin films Proc. NATO Adv. Res. Workshop (May 30-June 5, 1996) (NATO $A R W$ Series) ed Y Pauleau and P B Barna (Dordrecht: Kluwer) p 185

[12] Gardos M N and Soriano B L 1990 J. Mater. Res. 52599

[13] Gardos M N 1994 Synthetic diamond: emerging CVD science and technology Electrochemical Society Monograph ed K E Spear and J P Dismukes (New York: Wiley) ch 12, p 419

[14] Gardos M N and Ravi K V 1994 Diam. Films Technol. 4139

[15] Fijany A, Çağın T, Botero A J and Goddard III W A 1998 Novel algorithms for massively parallel, long term simulation of molecular dynamics systems Adv. Engng Soft. 29441

[16] Brenner D W 1990 Empirical potential for hydrocarbons for use in simulating the chemical vapor deposition of diamond films Phys. Rev. B 429458

[17] Che J, Çağın T and Goddard III W A Extenion of bond order dependent potentials to include long range interactions Theo. Chem. Acct. at press

[18] Che J, Çağın T and Goddard III W A 1999 Studies of fullerenes and carbon nanotubes by an extended bond order potential Nanotechnology 10263

[19] Walch S P, Goddard III W A and Çağın T 1999 Computational studies of the interaction of $\mathrm{H} / \mathrm{H} 2$ with diamond and silicon surfaces, to be published

[20] Çağın T and Che J 1998 Steady State Molecular Dynamics

[21] Cerius 1997 (San Diego, CA: Molecular Simulations Inc.)

[22] 1998 Cerius $^{2}$ Software Developers Kit (San Diego, CA: Molecular Simulations Inc.) 